Pacific Journal of Mathematics

FUNCTIONAL REPRESENTATION OF ALGEBRAIC 


\title{
FUNCTIONAL REPRESENTATION OF ALGEBRAIC INTERVALS
}

\author{
ROBERT E. JAMISON
}

\begin{abstract}
Motivated by some examples from the study of axiomatic convexity, we define a class of objects (in real algebras with 1) whose algebraic properties mimic those of the unit interval. These objects, called intervals, have quite a bit of structure in themselves. In particular, in a Banach algebra a compact interval must be finite dimensional. Even more striking is the main result which shows that any interval satisfying a very modest boundedness condition is commutative and can be represented by continuous functions from a compact Hausdorff space into the unit interval. This leads to a number of corollaries in analysis and topology.
\end{abstract}

THEOREM 1. Let $M$ be a linear space of real functions on a set $X$, and suppose that any function in $M$ is the difference of two nonnegative functions in $M$. If $S$ and $T$ are linear maps from $M$ to $M$ such that

$$
0 \leqq S(f) \leqq f \quad \text { and } \quad 0 \leqq T(f) \leqq f
$$

for all nonnegative $f$ in $M$, then $S$ and $T$ commute.

THEOREM 2. Let $\left\{a_{n}\right\}_{n=1}^{\infty}$ be a sequence of positive real numbers such that $\lim \sup \left(a_{n}\right)^{1 / n}=\infty$. Suppose $P$ is the smallest set of real polynomials containing 0 and $a_{n} x^{n}$, for all $n$, and satisfying

(1) $p(x) q(x) \in P$ if $p$ and $q$ are in $P$ and

(2) $-p(x)+1 \in P$ if $p$ is in $P$.

Then any real polynomial $r$ such that $0<r(0)<1$ is a convex combination of polynomials in $P$.

These two seemingly disparate results, as well as a representation theorem by Stone for partially ordered algebras, follow as natural corollaries of a general theory to be developed in this paper. This theory had its origins in an attempt to investigate more general notions of convexity in real linear spaces, and that setting still seems to provide the best starting place of the development.

Definition. If $A$ is a ring with 1 , then an interval in $A$ is a subset $I$ of $A$ such that

(1) $0 \in I$,

(2) $1-x \in I$ if $X \in I$, and 
(3) $x y \in I$ if $x$ and $y$ belong to $I$. If $M$ is a unitary left module over $A$, then a subset $C$ of $M$ is $I$-convex iff $x p+(1-x) q$ is in $C$ whenever $p$ and $q$ are points in $C$ and $x$ is in $I$.

The following properties, which resemble those of usual convex sets in real vector spaces, can be readily verified for the family of all $I$-convex subsets of $M$ :

(1) the arbitrary intersection of $I$-convex sets is again $I$-convex,

(2) the union of any chain of $I$-convex sets is again $I$-convex,

(3) if $C$ and $D$ are $I$-convex, then so is $C+D=\{p+q: p \in C$ and $q \in D\}$,

(4) if $C$ is $I$-convex and $r$ is an element of $A$ which centralizes $I$ (i.e., $r x=x r$ for all $x$ in $I$ ), then $r C$ is $I$-convex,

(5) every submodule of $M$ is $I$-convex,

(6) every singleton subset of $M$ is $I$-convex.

Since the ring $A$ is a module over itself, it is natural to ask whether a given interval $I$ is convex with respect to the convexity it induces on $A$. In other words, an interval $I$ is replete provided it satisfies the condition:

(4) $t x+(1-t) y \in I$ if $t, x$, and $y$ are in $I$. Although many common examples of intervals are replete, property (4) does not follow in general from the defining properties of an interval. As an illustration, let $J_{6}$ consist of 0 and 1 together with all rational numbers of the form $a / 6^{n}$ where $n$ is a positive integer and $0<a<6^{n}$ and $a \equiv \pm 1 \bmod 6$. Then $J_{6}$ is an (algebraic) interval of rational numbers, but it is not replete since

$$
(1 / 6)(1 / 6)+(1-1 / 6)(5 / 6)=26 / 36,
$$

which is not in $J_{6}$.

The product convexity defined on $\boldsymbol{R}^{n}$ by J. Eckhoff [4] provides an important example of an interval convexity. If $R^{n}$ is written as a direct sum of lower dimensional spaces, $\boldsymbol{R}^{n}=\boldsymbol{R}^{n_{1}}+\boldsymbol{R}^{n_{2}}+\cdots+\boldsymbol{R}^{n_{k}}$, then the product convexity is obtained by taking cartesian products of usual convex sets from each of the direct summands. Now $\boldsymbol{R}^{n}$ may be regarded as a module over its ring of linear endomorphisms $E\left(\boldsymbol{R}^{n}\right)$. If we let $I$ consist of all linear combinations in $E\left(\boldsymbol{R}^{n}\right)$ of the form

$$
\lambda_{1} P_{1}+\lambda_{2} P_{2}+\cdots+\lambda_{k} P_{k}
$$

where $P_{i}$ is the canonical projection of $\boldsymbol{R}^{n}$ onto $\boldsymbol{R}^{n_{i}}$ and $0 \leqq \lambda_{i} \leqq 1$, then $I$ is an interval in $E\left(\boldsymbol{R}^{n}\right)$. In fact, the Eckhoff product convexity coincides with the induced I-convexity.

Although many of the combinatorial properties of this product 
convexity have been studied [4, 14], our interest in intervals will be primarily algebraic and analytic. One might hope that other interesting convexities might arise from intervals. For example, what convexities are induced in a Banach space $B$ by norm compact intervals of bounded linear operators from $B$ to $B$ ? As we shall see, there are in fact very few. The defining conditions for an interval, although quite simply stated, are extremely powerful. In fact, in a real algebra with 1 any interval satisfying a modest boundedness condition is isomorphic to an interval of continuous real functions. The proof of this fact, together with a sharper structure theorem for compact intervals in Banach algebras, is the main objective of this investigation.

Throughout the remainder of this paper, $A$ will denote a real algebra with 1 , and, except when explicitly stated to the contrary, 'convex' will hereafter refer only to convexity in the usual vector space sense. Usually the emphasis will fall on convex intervals. However, this is only a mild restriction, for, as the reader may easily verify, the convex hull of an interval is again an interval.

ExAMPLES. Some further examples of intervals may be elucidating.

(I ) If $A$ is any algebra, the sets $I_{0}=\{0,1\}, I_{1}\{\lambda \cdot 1: 0 \leqq \lambda \leqq 1\}$, and $I_{\infty}=\{r \cdot 1: r \in R\}$ are all intervals. In any module $M$ over $A$, all subsets are $I_{0}$-convex, the $I_{1}$-convex sets coincide with sets convex in the usual sense (in $M$ as a real vector space), and the $I_{\infty}$-convex sets are the affine manifolds.

(II) If $A$ is an algebra of real functions on a set $X$, then the family of functions in $A$ which map $X$ into [0:1] is an interval. If $Y$ is any subset of $X$, then the family of all functions in $A$ mapping $X$ into [0:1] and taking only the values 0 and 1 on $Y$ is also an interval.

(IIa) In the special case where $X$ consists of just two points, $A$ may be visualized as $R^{2}$ with multiplication and addition defined coordinatewise. The square $\{(x, y): 0 \leqq x \leqq 1$ and $0 \leqq y \leqq 1\}$ and the strip $\{(x, y): 0<y<1\} \cup\{(0,0),(1,1)\}$ are two easily pictured examples that the reader may wish to keep in mind when encountering definitions and constructions introduced later.

(III) Let $M$ be a linear space of real functions on a set $X$, and let $A$ be an algebra of linear transformations of $M$ into $M$. The family of all transformations $T$ in $A$ such that

$$
0 \leqq T(f) \leqq f \text { for all } f \text { in } M \text { with } f \geqq 0
$$

constitutes an interval. The ordering here is, of course, pointwise. 
(IV) Suppose that $A$ is a partially ordered algebra [cf. 5, p. 105]. That is, $A$ possesses a partial order $\leqq$ such that

(1) $0 \leqq \lambda \cdot 1$ for all positive real $\lambda$,

(2) $x \leqq y$ implies $x+z \leqq y+z$ for all $x, y, z$ in $A$, and

(3) $x \leqq y$ implies $x z \leqq y z$ and $z x \leqq z y$ for all $x, y, z$ in $A$ with $0 \leqq z$.

Then the set of all $x$ in $A$ such that $0 \leqq x \leqq 1$ forms an interval.

(V) If $A$ is a commutative algebra, the set of all idempotents in $A$ is an interval.

As our investigation will center on intervals bounded in some way, the complex numbers will play no prominent role for the following reason: Any bounded interval $I$ in the complex field is, in fact, contained in the real unit interval. Clearly, if $I$ is bounded, the absolute value of any element of $I$ can be at most 1 . Now if $z$ is any complex number with $\operatorname{Re}(z)<0$, then $|1-z|>1$, so $I$ can contain no points in the open left half-plane. But if $z$ is any complex number with $\operatorname{Im}(z) \neq 0$, some power of $z$ lies in the left half-plane, so all numbers in $I$ must be real.

ExAmple (VI). Let $A$ be the Banach algebra $H_{\infty}(U)$ of all complex functions holomorphic and bounded on the open disc. If $I$ is a bounded interval in $A$, each point evaluation map sends $I$ into a bounded interval in the complex plane, and hence into the real unit interval. Since a real-valued holomorphic map must be constant, $I$ must consist of constant functions with values in [0:1].

The main result in historical perspective. As the means to a new proof of the Stone-Weierstrass theorem, R. I. Jewett obtained in a 1963 paper several beautiful results for intervals of continuous functions $[9,7, \mathrm{p} .101]$. (It should perhaps be noted that interest in intervals dates back at least to von Neumann [19, p. 93], who pointed out their connection with the theory of automata.) One consequence of Jewett's work is stated below.

THEOREM. (Jewett [9].) Suppose $X$ is a compact Hausdorff space and $I$ is an interval of continuous functions from $X$ into [0:1]. If the functions in I separate the points of $X$, and $I$ is uniformly closed, and $I$ contains the constant function $1 / 2$, then $I$ consists of all continuous functions from $X$ into $[0 ; 1]$.

There is also a theorem of Stone which has a connection with intervals. Recall that a partially ordered vector space $V$ is archimedean provided that, for any $x$ and $y$ in $V, n x \leqq y$ for all positive integers $n$ implies $x \leqq 0$. (This is equivalent to the requirement that the posi- 
tive cone $P=\{x \in V: x \geqq 0\}$ be linearly closed.) Also, recall that an element $e$ of $V$ is an order unit provided for each $x$ in $V$ there is some integer $m$ with $x \leqq m e$. [16, p. 205].

THEOREM. (Stone [17].) If $A$ is an archimedean partially ordered real algebra with 1 and 1 is an order unit, then $A$ is isomorphic to an algebra of continuous functions on a compact Hausdorff space.

Note that the commutativity of $A$ is a conclusion rather than a hypothesis. In fact, in the version of this theorem provided in $[10$, p. 7], even the associativity of $A$ is not assumed! Another proof is also available in Schaefer's book [16, p. 255]. (Actually, it might be appropriate to call the above result the Schaefer-Stone theorem, since it seems Stone never published a proof and the proof given by Schaefer in [16] is simpler and more general than what Stone suggests in [17].)

It should be noted in passing that Stone's theorem is but one of several results which conclude commutativity from order properties. One of the first is due to David Hilbert and appears in his celebrated Grundlagen der Geometrie [8, p. 105]. Some others may be found in [5, p. 145]. More recently, there is a commutativity result due to Dai and DeMarr [2] which, as will be shown later, actually follows rather easily from the Schaefer-Stone theorem.

Interval Representation Theorem. Suppose $I$ is a convex interval which generates the algebra $A$. If I is linearly closed and bounded (i.e., the intersection of $I$ with any line in $A$ is a closed segment), then $A$ is isomorphic to an algebra of continuous functions on a compact Hausdorff space $X$ and $I$ consists of all functions in a mapping $X$ into [0:1].

One might attempt to apply Stone's theorem to prove this by using the cone $P=\bigcup_{n=1}^{\infty} n I$ to order $A$. The hypotheses of Stone's theorem are easily verified - except the archimedean requirement. The archimedean condition is, in fact, satisfied, but this is by no means obvious a priori. It seems to rely on a rather subtle interplay between the geometry of the interval and its algebraic properties. A linearly bounded interval actually looks like a square or higher dimensional cube (see Example IIa). But it is not inconceivable that an interval might look like a circle, centered at $1 / 2$, with 0 and 1 in the circumference. In such a case, the cone generated would not be linearly closed and its linear closure would not be proper (since it would contain the tangent to the circle at 0 ). Ruling out the possibility of curvature of the boundary is the principal task in proving 
the representation theorem. Of course, even if one could use Stone's theorem immediately, one would not know that the order interval $\{x: 0 \leqq x \leqq 1\}$ coincided with the original interval $I$. Thus one would have to resort to different techniques to prove some analogue of Jewett's theorem for this case. At present, the difficulties concomitant with the possible curvature of an interval seem to be so great that a new approach, not utilizing the Schaefer-Stone theorem, appears necessary to prove the Interval Representation Theorem.

As with all representation theorems of this ilk, the compact Hausdorff space $X$ turns out to consist of multiplicative linear functionals on the algebra. There seem to be two general methods for obtaining these homomorphisms. One way is to examine an appropriately defined convex set of affine functions: in propitious circumstances the extreme points are multiplicative and exist in abundance $[1,10,16]$. The other attack, to be used here, is more internal. It involves the production of an ample supply of idempotents and maximal ideals. This has the advantage that it actually displays what is going on in the algebra.

Proposition 1. Let $I$ be a convex interal in an algebra $A$. Then

(a) every extremepoint of $I$ is an idempotent,

(b) an idempotent $e$ in $I$ is extreme iff 0 is an extremepoint of $I$,

(c) if I contains no nontrivial subspace of $A$, then 0 is an extremepoint of $I$.

Proof. (a) Let $t$ be an extremepoint of $I$. Then $2 t-t^{2}=$ $1-(1-t)^{2}$ is in $I$, and $t^{2}$ is in $I$, and

$$
t=\frac{1}{2}\left(2 t-t^{2}\right)+\frac{1}{2} t^{2},
$$

so $t^{2}=t$.

(b) Suppose first that 0 is an extremepoint and that $e+h$ and $e-h$ are in $I$ for some $h$. We must show that $h=0$. The roles of $h$ and $-h$ are symmetric, so whatever we prove for $h$ is also valid for $-h$ by a symmetric argument. First $e(1-(e-h))=e-e^{2}+$ $e h=e h$, since $e=e^{2}$. Thus $e h$ is in $I$. Similarly - eh is in $I$. Since 0 is extreme by hypothesis, $e h=0$. Analogous treatment of $(1-(e-$ $h)) e=$ he proves that $h e=0$. Next

$$
(e+h)(1-(e-h))=e-e^{2}+e h+h-h e+h^{2}=h+h^{2} .
$$

Thus $h+h^{2}$ is in $I$, but also 


$$
(e+h)(1-(e+h))=e-e^{2}-e h+h-h e-h^{2}=h-h^{2} .
$$

So $h-h^{2}$ is in $I$. Since $I$ is convex, we get

$$
h=\frac{1}{2}\left(h+h^{2}\right)+\frac{1}{2}\left(h-h^{2}\right)
$$

in $I$. Symmetrically, $-h$ is in $I$. Again, since 0 is an extremepoint, we must conclude that $h=0$.

For the converse, suppose that $e$ is an extreme idempotent of $I$ and both $h$ and $-h$ are in $I$. Again we must prove that $h=0$. Here $e+e h=e(1-(-h))$ and $e-e h=e(1-h)$ are in $I$. Since $e$ is extreme, we must have $e h=-e h=0$. Now

$$
1-[(1-e)(1+h)]=e-h+e h=e-h
$$

and

$$
1-[(1-e)(1-h)]=e+h-e h=e+h
$$

are both in $I$. Therefore, the extremeness of $e$ forces $h=0$.

(c) Again we must show that 0 is an extremepoint of $I$, so suppose $h$ and $-h$ are both in $I$ for some $h$. Then $h+h^{2}=h(1-$ $(-h))$ is in $I$ as is $2 h-h^{2}=1-(1-h)^{2}$. By convexity of $I$, we find that

$$
\frac{3}{2} h=\frac{1}{2}\left(2 h-h^{2}\right)+\frac{1}{2}\left(h+h^{2}\right)
$$

is in $I$. Symmetrically, $3 / 2(-h)$ is also in $I$. Thus we may repeat the argument $a d$ infinitum to get the whole line thru $h$ and 0 in $I$. But then this line is a subspace contained in $I$, so $h$ must in fact be 0 .

Proposition 2. If $I$ is a interval in an algebra $A$ and $I$ contains no rays from 0 (i.e., $n x \in I$ for all positive integers $n$ implies $x=0$ ), then

(a) I contains no nonzero nilpotents,

(b) if $e$ is an idempotent in $I$, then ex $=x e$ for all $x$ in $I$.

Proof. (a) Suppose $s$ is a nonzero nilpotent in $I$. Then for some $k>0, s^{k} \neq 0$, and $s^{k+1}=0$. Let $t=s^{k}$. Then $t \neq 0, t \in I$, and $t^{2}=0$. Now observe that $n t=1-(1-t)^{n}$ is in $I$ for all $n$, contrary to our assumption.

(b) Consider the element $e x-e x e=e x(1-e)$, which is in $I$. Then, since $e$ is idempotent,

$$
(e x-e x e)^{2}=0 .
$$


By part (a), this forces $e x-e x e=0$, so $e x=e x e$. Now a similar treatment of $x e-e x e=(1-e) x e$, which is in $I$, yields $x e=e x e$. Hence $e x=x e$.

We shall mean by a topological algebra an algebra equipped with a linear space topology such that, for each $p$ in $A$, the maps $x \mapsto x p$ and $x \mapsto p x$ are continuous. All topologies given consideration are assumed to be Hausdorff. By a normed algebra we shall mean an algebra equipped with a linear space norm $\|\cdot\|$ such that $\|x y\| \leqq$ $\|x\|\|y\|$ and $\|1\|=1$. The standard analysis theorems to be used are available in such references as $[3,13,16,18]$.

It is convenient to state here a simple but useful lemma which seems to be known, in various guises, as a folk theorem. (Note that the proof does not really require associativity.)

Lemma A. Let $X$ be a topological space on which a semigroup operation $(x, y) \mapsto x y$ is defined such that, for each $q$ in $X, x \mapsto x q$ is continuous. Suppose $Y$ is a subsemigroup of $X$ and that, for each $p$ in $Y$, the map $x \rightarrow p x$ is continuous from $X$ into $X$. Then

(i) $\operatorname{cl} Y$ is a subsemigroup of $X$, and

(ii) if cl $Y$ contains a dense central subset $Z$, then $Y$ is central in cl $Y$ and, in particular, $Y$ is commutative.

Proof. (i) If $p$ and $q$ are in cl $Y$, there are nets $p_{\gamma}$ and $q_{\eta}$ in $Y$ converging, respectively, to $p$ and $q$. Now for fixed $\gamma, p_{\gamma} q$ is the limit over $\eta$ of $p_{\gamma} q_{\eta}$, since $p_{\gamma}$ is in $Y$. Hence each $p_{\gamma} q$ is in $\mathrm{cl} Y$, so taking the limit over $\gamma$, we get $p q$ in cl $Y$.

(ii) Let $p \in \operatorname{cl} Y$ and $y \in Y$. Select a net $z_{\gamma}$ in $Z$ with $z_{\gamma}$ converging to $p$. Then $p y$ is the limit of $z_{\gamma} y$. Since $z_{\gamma}$ is central, this is the same as the limit of $y z_{r}$, which, because $y \in Y$, equals $y p$.

THeORem A. If $I$ is a compact convex interval in a locally convex topological algebra $A$, then $I$ is commutative. If, in addition, 0 is not a limit point of nonzero idempotents, then there is a finite set $\left\{e_{i}\right\}_{i=1}^{n}$ of orthogonal idempotents such that I consists of all points of the form

$$
\sum_{i=1}^{n} \lambda_{i} e_{i} \quad \text { where } \lambda_{i} \in[0: 1] \text { for each } i .
$$

Proof. Since $I$ is compact, it satisfies the conditions for both propositions. Hence the set $E$ of idempotents in $I$ coincides with the set of extreme points of $I$, and $E$ is central in $I$. Clearly conv $E$ is also central in $I$, and by the Krein-Milman theorem $I=\mathrm{cl}$ conv $E$. Applying Lemma A with $X=A, Y=I$, and $Z=\operatorname{conv} E$, we may 
conclude that $I$ is commutative.

Let us now suppose that 0 is not the limit of nonzero idempotents. Since $E$ is commutative it can be given the natural partial order: $f \leqq e$ iff $e f=f[7$, p. 11]. Let $C$ be a decreasing chain of nonzero idempotents in $I$. Directed by the natural partial order, $C$ is a net and hence has a convergent subnet $e_{\gamma} \rightarrow f$. For any $g$ in $C, e_{\gamma}$ is eventually less than $g$, so $g f=\lim g e_{\gamma}=\lim e_{\gamma}=f$. Thus since each $e_{\gamma}$ is in $C, f^{2}=\lim e_{\gamma} f=\lim f=f$. Whence $f$ is an idempotent less than all $g$ in $C$, and $f \neq 0$ by the hypothesis of the theorem. Adducing Zorn's lemma, we may conclude that, given any nonzero idempotent $e$ in $I$, there is a minimal nonzero idempotent $f$ in $I$ with $f \leqq e$.

We now claim that the set $E_{m}$ of minimal nonzero idempotents is finite. If not, there would be a net $e_{r}$ in $E_{m}$ converging to some $f$ with $e_{\gamma} \neq f$ for all $\gamma$. For any chosen $e_{0}$ in the net, $e_{0} f$ is the limit over $\gamma$ of $e_{0} e_{\gamma}$. Since $e_{0} \neq f$, eventually $e_{\gamma}$ is different from $e_{0}$. But distinct minimal idempotents are orthogonal, so $e_{0} e_{\gamma}$ is eventually 0 . Thus $e_{0} f=0$ for each $e_{0}$ in the net. Thus $f^{2}=\lim e_{\gamma} f=0$. By Proposition 2a, $f$ is zero, contrary to hypothesis. Hence $E_{m}$ is finite.

Note that if $x$ and $y$ are in $I$ and $x y=0$, then $x+y=1-$ $(1-x)(1-y)$ is in $I$. Applying this inductively, we may conclude that the sum $s$ of the minimal nonzero idempotents is in $I$. Clearly $s$ is idempotent. If $s \neq 1$, there is an $f$ in $E_{m}$ less than $1-s$. It follows from the definition of $s$ that $s f=f$. But $f \leqq 1-s$ means $(1-s) f=f$, so $f=0$, a contradiction. Hence 1 is the sum of the minimal nonzero idempotents.

For any two idempotents $e$ and $f$, ef $\leqq f$, so, if $f$ is minimal, either $e f=f$ or $e f=0$. Thus, for any idempotent $e$ in $E, e=e \cdot 1=$ $\Sigma\left\{f \in E_{m}: f \leqq e\right\}$. Whence $E$ is finite. It follows that conv $E$ is already closed, so $I=\operatorname{conv} E$.

Now, using the facts that any point of $I$ is a convex combination of idempotents in $E$ and any idempotent is the sum of minimal idempotents, it is easy to write any point of $I$ in the form $\left(^{*}\right)$, where $\left\{e_{i}\right\}_{i=1}^{n}=E_{m}$. Conversely, the orthogonality of the minimal idempotents implies that any point of the form $(*)$ is in $I$.

In a Banach algebra, a nonzero idempotent has norm at least 1 , so Theorem A settles the question about convexities induced on a Banach space $B$ by a compact interval of operators on $B$. The idempotents $e_{i}$ are, of course, projections in this case, so $B$ can be written as the topological sum of their ranges - in analogy to the Eckhoff convexities in $\boldsymbol{R}^{n}$.

EXAMPLE (VII). Let $A$ be the algebra of all bounded real functions on [0:1] with the topology of pointwise convergence. The interval $I$ of all functions from [0:1] into [0:1] is a compact convex 
interval in $A$, but it is not finite dimensional. Let $e_{n}$ be the characteristic function of $(0: 1 / n)$. Each $e_{n}$ is idempotent and $\lim _{n \rightarrow \infty} e_{n}=0$.

Scholium. Let $A$ be a normed algebra and $M$ the set of all continuous homomorphisms of $A$ into the complex numbers $C$. Suppose $I$ is a norm bounded interval in $A$. If $\phi$ and $\psi$ belong to the same connected component of $M$ with respect to the strong (norm) topology on $M$, then $\phi(x)=\psi(x)$ for all $x$ in $I$.

Proof. We may suppose that $I$ is convex, since the convex hull of a bounded interval is again a bounded interval. Now let $b$ be a bound on the norms of elements of $I$. We shall show that if $\|\phi-\psi\|<$ $1 / b$, then $\phi(x)=\psi(x)$ for all $x$ in $I$. The result will then follow by a routine connectivity argument.

To check the claim, suppose $\phi(p) \neq \psi(p)$ for some $p$ in $I$ where $\phi$ and $\psi$ are in $M$. The map $x \rightarrow(\phi(x), \psi(x))$ is a continuous homomorphism of $A$ into $C^{2}$, where addition and multiplication are coordinatewise. The image of $I$ under this homomorphism is then a bounded convex interval $J$. The closure of $J$ is thus a compact interval and hence is the convex hull of its idempotents. Since $\phi(p) \neq \psi(p)$, cl $J$ cannot be the segment from $(0,0)$ to $(1,1)$; it must be the square whose vertices are the four idempotents in $\boldsymbol{C}^{2}$. Since $J$ is dense in this square, for any $\varepsilon>0$, one can find a point $x$ of $I$ with $\phi(x)>1-\varepsilon$ and $\psi(x)<\varepsilon$. Thus

$$
\|\phi-\psi\| \geqq\|\phi(x)-\psi(x)\| /\|x\| \geqq(1-2 \varepsilon) / b .
$$

Since $\varepsilon$ is arbitrary, this finishes the proof.

EXAMPLE (VIII). Let $X$ be a compact connected metric space with metric $d$. Let $\operatorname{Lip}(X)$ denote the space of all real-valued functions on $X$ which satisfy a Lipschitz condition of order 1 [7, p. 270]. Then $\operatorname{Lip}(X)$ is a Banach algebra with the norm given by

$$
\begin{aligned}
\|f\|= & \sup _{x \in X}|f(x)|+\inf \{\lambda:|f(x)-f(y)| \leqq \lambda d(x, y) \\
& \text { for all } x \text { and } y \text { in } X\} .
\end{aligned}
$$

We claim that any norm bounded interval in Lip $(X)$ consists entirely of constants. Note that each point $x$ in $X$ induces a continuous homomorphism $f \rightarrow f(x)$. The norm topology on these evaluation homomorphisms agrees with the original metric topology on $X$. Thus the above claim is just a special case of the scholium.

MaIN Lemma. If I is a norm closed and bounded convex interval 
in a normed algebra $A$, then $I$ is commutative and replete.

Proof. Let $A^{*}$ be the topological dual of $A$. For each $y$ of $A$, let $T_{y}$ be the operator from $A^{*}$ to $A^{*}$ whose action on an element $f$ of $A^{*}$ is defined by

$$
\left(T_{y} f\right)(x)=f(x y) \text { for all } x \text { in } A .
$$

Thus $T_{y}$ is the adjoint of right multiplication by $y$ [cf. 15, p. 66]. Hence the map $y \rightarrow T_{y}$ is an algebra isomorphism of $A$ into the (complete) normed algebra $B$ of all norm continuous linear transformations of $A^{*}$ into $A^{*}$. We check quickly that this map is also an isometry:

$$
\left\|T_{y}(f)\right\|=\sup _{\|x\|=1}|f(x y)| \leqq \sup _{\|x\|=1}\|f\|\|x\|\|y\|=\|f\|\|y\| .
$$

Thus $\left\|T_{y}\right\| \leqq\|y\|$. For the reverse inequality, choose by the HahnBanach theorem a functional $g$ on $A$ with $\|g\|=1$ and $|g(y)|=\|y\|$. Then since $g \in A^{*}$,

$$
\left\|T_{y}\right\|=\sup _{\|f\|=1}\left\|T_{y}(f)\right\| \geqq\left\|T_{y}(g)\right\|=\sup _{\|x\|=1}|g(x y)| \geqq|g(1 \cdot y)|=\|y\| .
$$

Let us put on $B$ the weak* operator topology, whose subbasic neighborhoods of the origin are of the form

$$
N(f ; U)=\{T \in B: T(f) \in U\}
$$

where $f \in A^{*}$ and $U$ is a weak* neighborhood of 0 in $A^{*}$. A net $\left(T_{r}\right)$ of operators in $B$ converges to an operator $T$ in this weak* operator topology if and only if $T_{\gamma}(f) \rightarrow T(f)$ in the weak* topology on $A^{*}$ for all $f$ in $A^{*}$. It is clear that this topology is a locally convex linear topology on $B$, but unfortunately multiplication may fail to be simultaneously continuous in both variables.

However, there is enough continuity for the advancement of the argument. For any fixed $S$ in $B$, the map $T \rightarrow T \circ S$ is weak* operator continuous. For if $T_{\gamma} \rightarrow T$ and $f \in A^{*}$, then $T_{\gamma}(S(f)) \rightarrow T(S(f))$. As a partial dual, if $S$ is weak* continuous from $A^{*}$ into $A^{*}$ as well as being norm continuous, then the map $T \rightarrow S \circ T$ is weak* operator continuous. For if $T_{r} \rightarrow T$ and $f \in A^{*}$, then $T_{r}(f) \rightarrow T(f)$ so, by continuity of $S, S\left(T_{r}(f)\right) \rightarrow S(T(f))$.

It is fortunate that $T_{y}$ is weak* continuous from $A^{*}$ into itself for every $y$ in $A$. To see this, it suffices to observe that the preimage under $T_{y}$ of any subbasic weak* open set is again weak* open in $A^{*}$. But if $U=\left\{f \in A^{*}: f(x) \in V\right\}$, where $x$ is some point in $A$ and $V$ is open in $R$, then $\left\{g \in A^{*}: g(x y) \in V\right\}$ is the preimage of $U$ under $T_{y}$ and is weak* open. 
Alaoglu's theorem asserts that the unit ball $D$ of $A^{*}$ is weak* compact [13, p. 155]. By imitating the proof of this theorem, one can readily show that the unit ball $K$ of $B$, which consists of all linear operators from $A^{*}$ into $A^{*}$ sending $D$ into $D$, is compact in the weak* operator topology.

The image $J$ of $I$ under the embedding $y \mapsto T_{y}$ is a convex interval in $B$. Since $I$ is norm bounded and the embedding is an isometry, $J$ is contained in some multiple $n K$ of $K$. But $n K$ is weak* operator compact, so the weak* operator closure $H$ of $J$ is also compact. Setting $X=B$ and $Y=J$, we see from Lemma A that $H$ is a semigroup. The other requirements for a convex interval follow easily, so $H$ is a compact convex interval.

Applying the Krein-Milman theorem in conjunction with Proposition 1, we see that $H$ is the closed convex hull of its set $E$ of idempotents. Proposition 2 implies that $E$, and hence conv $E$, is central in $H$. Using Lemma A again with $X=B, Y=J$, and $Z=$ conv $E$, we see that $J$, and hence $I$, is commutative.

Let us now show that $I$ is replete. First, since the idempotents in $E$ commute, $E$ is a semigroup and, in fact, an interval. Actually $E$ is replete. For if $e, f$, and $g$ are in $E$, then $(e f)(1-e) g=0$ so that (as in the proof of Theorem A) the sum ef $+(1-e) g$ must belong to the interval $E$. Exercising a little care, it is not too difficult to convince oneself that the convex hull of a replete interval is replete. Thus conv $E$ is also a replete interval.

Since the idempotents in $E$ commute with everything in $H$, composition of elements of $H$ from either side with a fixed element of conv $E$ is continuous. Now if $R, S$, and $T$ are operators in $H$, there are nets of operators in conv $E$ such that $R_{\gamma} \rightarrow R, S_{\eta} \rightarrow S$, and $T_{0} \rightarrow T$. For fixed $\gamma$, conv $E$ contains $R_{\gamma} \circ S_{\eta}+\left(1-R_{\gamma}\right) \circ T_{\theta}$ for all $\eta$ and $\theta$. (Here 1 denotes the identity operator.) Since addition is jointly continuous, the double net indexed by $\eta, \theta$ converges to $R_{r} \circ S+$ $\left(1-R_{\gamma}\right) \circ T$, which must, therefore, belong to $H$. Now taking limits over $\gamma$ demonstrates the repleteness of $H$.

Now let $x, y$, and $z$ belong to $I$. Then $T_{x y+(1-x) z}=T_{x} \circ T_{y}+$ $\left(1-T_{x}\right) \circ T_{z}$ belongs to $H$ and is, therefore, the weak* operator limit of elements from $J$, the canonical image of $I$. We claim that this forces $x y+(1-x) z$ to be in $I$, thereby proving that $I$ is replete. To verify this, it suffices to show that if $w$ is any point of $A$ not in $I$, then $T_{w}$ is not the weak* operator limit of a net in $J$.

Assuming the contrary, suppose $T_{x(r)} \rightarrow T_{w}$ where each $x(\gamma)$ is in $I$. Since $I$ is convex and norm closed and $w \notin I$, there is a continuous linear functional $f$ on $A$ with $f(w)>\sup f[I][16$, p. 65]. For this $f$, we must have $T_{x(r)}(f) \rightarrow T_{w}(f)$ in the weak* topology on $A^{*}$. In particular, at the point 1 of $A, T_{x(r)}(f)(1) \rightarrow T_{w}(f)(1)$. 
Evaluating, this is $f(1 \cdot x(\gamma)) \rightarrow f(1 \cdot w)$, violating the choice of $f$. Thus the claim is valid, and the lemma is proved.

The next step toward the representation theorem is to show that, under suitable boundedness conditions a convex interval induces an algebra norm on the algebra it generates. Then the Main Lemma and the theory of commutative normed algebras may be applied. As a measure of boundedness, we associate with a convex interval $I$ in an algebra $A$ the set $I^{\prime}=\{t \in A: 1 / 2+n t \in I$ for all integers $n>0\}$. Because of the convexity and symmetry of $I$ about $1 / 2, I^{\prime}$ may be regarded geometrically as the set of all directions in which $I$ is unbounded. Algebraically, $I^{\prime}$ is the largest linear subspace of $A$ contained in $I-1 / 2 \cdot 1$. Thus $I^{\prime}=0$ iff $I$ is linearly bounded (i.e., the intersection of $I$ with any line is a bounded subset of that line). Algebraically $I^{\prime}$ behaves like an "unboundedness radical" - that is, it is an ideal and, when factored out, yields a linearly bounded interval.

Proposition 3. Let $I$ be a convex interval in an algebra $A$.

(a) The subalgebra $A(I)$ generated by $I$ in $A$ is given by $A(I)=\{m x-n \cdot 1: x \in I$ and $m$ and $n$ are nonnegative integers $\}$.

(b) $I$ is radial from $1 / 2$ in $A(I)$.

(c) The set $I^{\prime}=\{t \in A: 1 / 2+n t \in I$ for all positive integers $n\}$ is an ideal in $A(I)$.

(d) If $x \in I$ and $x^{-1}$ exists in $A(I)$, then $x^{-1}=m y$ for some $y$ in $I$ and positive integer $m$.

Proof. (a) Let $P=\{m x: x \in I$ and $m$ is a nonnegative integer $\}$. Then $P$ is a convex cone since $I$ is convex, and $P$ is a semigroup since $I$ is a semigroup. The linear span of $P$ is $P-P$, and this, as the span of a semigroup, must be an algebra. $P-P$ is therefore the algebra generated by $I$. But if $m x-n y$ is in $P-P$ where $x$ and $y$ are in $I$ and $m$ and $n$ are nonnegative integers (not both 0 ), then

$$
m x-n y=(m+n)\left[\left(\frac{m}{m+n}\right) x+\left(\frac{n}{m+n}\right)(1-y)\right]-n \cdot 1
$$

is the desired form.

(b) For any $x$ in $I, 1 / 2 \cdot 1+1 / 2 x$ is in $I$, so $I$ contains a small segment in the direction of $x$ with endpoint at $1 / 2$. Clearly $I$ is also radial from $1 / 2$ in the direction of -1 . But the set of directions in which a convex set is radial from a fixed point is a convex cone, and by (a) the smallest convex cone containing $I$ and -1 is all of $A(I)$. (c) The relation $1 / 2-n t=1-(1 / 2+n t)$ shows $I^{\prime}=-I^{\prime}$. The convexity of $I^{\prime}$ follows immediately from the convexity of $I$. But 
$I^{\prime}$ is certainly closed under multiplication by positive integers; hence $I^{\prime}$ is a linear subspace. For any $x$ in $I$, the following average is in $I$ :

$$
\frac{1}{2}+n t x=\frac{1}{2}\left(1-\frac{1}{2} x\right)+\frac{1}{2}\left(\frac{1}{2}+2 n t\right) x .
$$

A similar relation on the left finishes the proof that $I^{\prime}$ is an ideal in the algebra generated by $I$.

(d) Since $x^{-1}$ is in $A(I)$, by (a) it can be written as $x^{-1}=m y-n$ where $m$ and $n$ are nonnegative integers and $y$ is in $I$. If $n \neq 0$, we shall show that we can find another representation of $x^{-1}$ involving $n-1$, and thus after a finite number of reductions $x^{-1}$ can be put in the required form.

Note that $1=x(m y-n)=m x y-n x$, so (*) $m x y=n x+1$. Thus

$$
\begin{aligned}
x^{-1} & =m y-n\left(x \cdot x^{-1}\right)=m y-n x(m y-n) \\
& =m y-n m x y+n^{2} x=m y-m x y+(1-n) m x y+n^{2} x \\
& =m(1-x) y+(1-n)(n x+1)+n^{2} x \text { by }\left(^{*}\right) \\
& =m(1-x) y+n x+1-n^{2} x-n+n^{2} x . \\
& =m(1-x) y+n x-(n-1) \\
& =(m+n)\left[\frac{m}{m+n}(1-x) y+\frac{n}{m+n} x\right]-(n-1) .
\end{aligned}
$$

In order to obtain the crucial norm for the proof of the interval representation theorem, it is necessary to introduce a somewhat less natural auxillary norm. NoTE: For the next lemma and pair of propositions, it is assumed that $I$ is a convex, linearly closed interval which generates the algebra $A$ and that $I^{\prime}=0$.

Lemma B. Let $\|\cdot\|_{I}$ be the Minkowski gauge functional on $A$ determined by conv $(I \cup-I)$. Then $\|\cdot\|_{I}$ is an algebra norm on $A-$ that is, $\|x y\|_{I} \leqq\|x\|_{I} \cdot\|y\|_{I}$ and $\|1\|_{I}=1$.

Proof. Let $U=\{2 x-1: x \in I\}$. Then $U$ is an affine image of $I$, and $1 / 2$ in $I$ corresponds to 0 in $U$. Since $I^{\prime}=0$ means (geometrically) that $I$ contains no rays from $1 / 2, U$ contains no rays from 0 . Thus the Minkowski guage functional $|\cdot|_{I}$ associated with $U$ vanishes only at 0 . Since $I$ is radical from $1 / 2$ by Proposition $3 \mathrm{~b}, U$ is absorbing, so $|\cdot|_{I}$ is finite-valued. Hence $|\cdot|_{I}$ is a bona fide linear space norm on $A$.

But conv $(I \cup-I) \subseteq U$ since, for any $x$ in $I, x=2((1 / 2) x+1 / 2)-1$ and $-x=2(1 / 2(1-x))-1$. Thus $\|\cdot\|_{I} \geqq|\cdot|_{I}$. Also $(1 / 2) U=(1 / 2) I+$ 
$1 / 2(-1) \leqq \operatorname{conv}(I \cup-I)$, so $2|\cdot|_{I} \geqq\|\cdot\|_{I}$. Hence $\|\cdot\|_{I}$ is an equivalent linear space norm on $A$. It remains only to show that $\|\cdot\|_{I}$ is an algebra norm. Since $I$ is a semigroup, $I U-I$ is a semigroup, and hence its convex hull is a semigroup. Thus $\|\cdot\|_{I}$ is the gauge function of a convex semigroup and, therefore, satisfies the multiplicative inequality. It is now trivial to check that $\|1\|_{I}=1$, so $\|\cdot\|_{I}$ is a true algebra norm and the lemma is proved.

Proposition 4. Let $U=\{2 x-1: x \in I\}$, and let $|\cdot|_{I}$ be the Minkowski gauge on $A$ associated with $U$. Then

(a) $I$ is replete and $A$ is commutative.

(b) $|\cdot|_{I}$ is an algebra norm on $A$.

Proof. (a) Since $U$ contains $\operatorname{conv}(I \cup-I), 0$ is in the $\|\cdot\|_{I}$ interior of $U$. Thus $1 / 2$ is in the interior of $(1 / 2) U+1 / 2=I$. In any topological vector space, the linear closure and topological closure of any convex set with interior coincide [18, p. 13]. Because we made the standing hypothesis that $I$ is linearly closed, $I$ is also $\|\cdot\|_{I}$ - closed. Since $\|\cdot\|_{I}$ is an algebra norm, we may adduce the Main Lemma to conclude (a).

(b) It was already observed in the proof of Lemma B that $|\cdot|_{I}$ is a linear space norm. Trivially $|1|_{I}=1$. Hence the critical matter here is proving the multiplicative inequality - that is, showing that $U$ is a semigroup. This utilizes the repleteness of $I$.

If $p=2 x-1$ and $q=2 y-1$ lie in $U$, then

$$
p q=(2 x-1)(2 y-1)=2(2 x y-x-y+1)-1 .
$$

Since $x$ and $y$ are in $I$, by repleteness, $I$ also contains

$$
1-[x(1-y)+(1-x) y]=2 x y-x-y+1 .
$$

This is just the form needed to show that $p q$ lies in $U$. Hence $U$ is a semigroup, and the proposition is established.

Proposition 5. Let $M_{I}=\{x \in I: x+\lambda \cdot 1 \notin I$ for any $\lambda>0\}$. Then

(a) $M_{I}=\{x \in I: \mu x \notin I$ for any $\mu>1\}$,

(b) if $x$ belongs to $M_{I}$, then $1-x$ is singular in $A$,

(c) if $|p|_{I}=1$, then, for some $x$ in $M_{I}$, either $p=2 x-1$ or $-p=2 x-1$.

Proof. (a) Suppose $x$ and $x+\lambda$ both belong to $I$ for some $\lambda>0$. Then since $I$ is replete, $x(x+\lambda)+(1-x) x=(1+\lambda) x$ must belong to $I$ and $1+\lambda>1$.

Conversely, if $I$ contains both $x$ and $\mu x$ for some $\mu>1$, then 


$$
\frac{1}{\mu}(\mu x)+\left(1-\frac{1}{\mu}\right) \cdot 1=x+(\mu-1) / \mu
$$

must belong to $I$, and $(\mu-1) / \mu>0$.

(b) If $x$ is in $I$ and $1-x$ is regular in $A$, then by Proposition $3 \mathrm{~d}$ there is a $y$ in $I$ with $(1-x)^{-1}=m y$ for some $m>0$. That is, $y(1-x)=1 / m$. Whence $I$ must contain $1-(1-y)(1-x)=x+1 / m$, so $x$ is not in $M_{I}$.

(c) If $x$ is in the boundary of $I$, we claim that either (i) for any $\mu>1, \mu x \notin I$ or (ii) for any $\mu>1, \mu(1-x) \notin I$. Supposing (i) and (ii) both fail, there is then a $\mu>1$ such that $I$ contains both $\mu x$ and $\mu(1-x)$. Thus $I$ also contains the point

$$
y=\frac{1}{2}(\mu x)+\frac{1}{2}(1-\mu(1-x))=\mu\left(x-\frac{1}{2}\right)+\frac{1}{2} .
$$

Since $\mu>1, x$ lies in the relative interior of the line segment from $y$ to $1 / 2$. Because $1 / 2$ is in the interior of $I$, this impels $x$ to lie in the interior of $I$ as well [18, p. 9]. Hence the claim is true.

The affine map $x \rightarrow 2 x-1$ carries the boundary of $I$ onto the boundary of $U$. Thus if $|p|_{I}=1$, we can write $p=2 x-1$ where $x$ is in the boundary of $I$. If $x$ satisfies (i), then $x \in M_{I}$ and we are done. If $x$ satisfies (ii), then $1-x \in M_{I}$ and $-p=2(1-x)-1$ is the required form.

Although, in light of the Main Lemma, the equivalence of the two definitions of $M_{I}$ may seem rather trivial, this equivalence is actually the key point of the argument. The reader may wish to check heuristically (by making some plausible sketches in the plane) that the algebraic information of Proposition 5a effectively eliminates the possibility of curvature of the boundary of $I$.

The stage is now set for the final step in the proof of the representation theorem. Whenever $A$ is a real algebra with 1 and $S$ is a subset of $A$ that generates $A$ as an algebra, then the carrier of $S$ is defined as the set of all nontrivial homomorphisms $\phi$ of $A$ into $R$ such that $\phi[S] \subseteq[0: 1]$. Of course, the carrier of $S$ may be empty, but when it is nonempty, it can be given the topology of pointwise convergence on $S$. Since $S$ generates $A$, pointwise convergence of homomorphisms on $S$ implies pointwise convergence on all of $A$. Thus the carrier $\Phi$ of $S$ may be regarded as a closed subset of the compact space [0:1] $]^{S}$. Also when the carrier $\Phi$ of $S$ is nonempty, there is a natural homomorphism $\Pi_{S}$ from $A$ into $C(\Phi)$ given by

$$
\Pi_{S}(x)(\phi)=\phi(x) \text { for all } x \in A, \phi \in \Phi \text {. }
$$


Here $C(\Phi)$ denotes the algebra of all real-valued continuous functions on $\Phi$.

THeOREM B. Suppose that I is a linearly closed and bounded $\left(I^{\prime}=0\right)$ convex interval that generates an algebra $A$. Then the carrier $\Phi$ of $I$ is nonempty and the $\operatorname{map} \Pi_{I}: A \rightarrow C(\Phi)$ is an isometric isomorphism of $A$ under the norm $|\cdot|_{I}$ into $C(\Phi)$ under the supremum norm. Also, $\Pi_{I}[I]$ consist of all functions in $\Pi_{I}[A]$ which $\operatorname{map} \Phi$ into $[0: 1]$.

Proof. We take first the case in which $A$ is a Banach algebra under $|\cdot|_{I}$. (The reader may wish to check as an aside that this happens precisely when $I$ is complete with respect to the metric induced by $|\cdot|_{I}$.)

Let us show that any singular element $x$ of $A$ is mapped to 0 by some homomorphism $\phi$ in $\Phi$. By Proposition $4 \mathrm{a}, A$ is commutative, so $x A$ is a proper ideal in $A$. Thus $x A$ is contained in a maximal ideal $M$ of $A$. The quotient algebra $A / M$ is a field and can be given the quotient norm since $M$ is closed. (Recall that in a Banach algebra, the units form an open set, so all maximal ideals are closed [15, p. 12].) Thus by the Gelfand-Mazur theorem, $A / M$ is either $C$ or $\boldsymbol{R}$. Because its range is finite dimensional over $R$ and its null space $M$ is closed, the quotient map $\phi: A \rightarrow A / M$ is continuous as a real linear transformation. Therefore, $\phi$ maps the $|\cdot|_{I}$-bounded convex interval $I$ into a bounded convex interval $\phi[I]$ in $A / M$. In view of the remarks prior to Example VI, $\phi[I]$ must actually be the real unit interval. Thus $\phi$ is in the carrier $\Phi$ of $I$ and $\phi(x)=0$ (since $x \in x A \subseteq M$ ). In particular, we now know $\Phi$ is nonempty since zero is singular.

That $\Pi_{I}$ is an isometry is now an easy consequence of Proposition 5. Since the $|\cdot|_{I}$-unit ball of $A$ is the set $2 I-1$, it is easily seen that $\Phi$ consists of just those real-valued homomorphisms $\phi$ of $A$ with $\|\phi\| \leqq 1$. Therefore, $|\phi(p)| \leqq|p|_{I}$ for all $p$ in $A$ and all $\phi$ in $\Phi$, so $\left\|\Pi_{I}(p)\right\|_{\text {unif }} \leqq|p|_{I}$ where $\|\cdot\|_{\text {unif }}$ denotes the supremum norm on $C(\Phi)$. It suffices to verify the reverse inequality when $|p|_{I}=1$. Under that assumption, Proposition 5c implies that $p=$ $2 x-1$ or $-p=2 x-1$ where $x$ is in $M_{I}$. Now $1-x$ is singular by Proposition 5b, so, as shown above, there is a $\phi$ in $\Phi$ with $\phi(1-x)$ $=0$. For this $\phi, \phi(p)= \pm 1$; whence $\left\|\Pi_{I}(p)\right\|_{\text {unif }} \geqq 1=|p|_{I}$.

It remains to identify $\Pi_{I}[I]$. Since $A$ is complete, the StoneWeierstrass theorem implies that $\Pi_{I}[A]$ must be all of $C(\Phi)$. Now $U$ is the unit ball of $A$, so $\Pi_{I}[U]$ consists of all continuous functions mapping $\Phi$ into [-1:1]. From the definition of $U$, any point $x$ of $I$ can be written as $x=1 / 2(p+1)$ where $p$ is in $U$. Thus $\pi_{I}[I]$ consists of all continuous functions mapping $\Phi$ into [0:1]. This finishes the 
case in which $A$ is complete.

We now advance to the general case. Since $|\cdot|_{I}$ is an algebra norm, the completion $\bar{A}$ of $A$ with respect to $|\cdot|_{I}$ is a Banach algebra. Let $J$ denote the closure of $I$ in $\bar{A}$. Then $J$ is a closed convex interval and the set $\bar{U}=\{2 x-1: x \in J\}$ is the closure in $\bar{A}$ of $U=$ $\{2 x-1: x \in I\}$. Since $U$ is the unit ball in $A, \bar{U}$ must be the unit ball of $\bar{A}$, so the norm on $\bar{A}$ is none other than $|\cdot|_{J}$. Whence we are back in the complete case.

Letting $\Psi$ denote the carrier of $J$, the argument for the complete case demonstrates that $\Pi_{J}$ is an isometry of $\bar{A}$ onto $C(\Psi)$. Now $I$ is norm-closed in $A$. ( $I$ is linearly closed and has nonvoid $|\cdot|_{I}$-interior in $A$.) Thus $I=J \cap A$, so $\Pi_{J}[I]$ consists of all functions in $\Pi_{J}[A]$ which send $\Psi$ into [0:1].

The proof will be finished if it can be shown that $\Psi$ and the carrier $\Phi$ of $I$ in $A$ are really the same (under a suitable identification). In fact, we claim that each $\bar{\phi}$ in $\Psi$ is the extension of a unique $\phi$ in $\Phi$. Surely the restriction of any $\bar{\phi}$ to $A$ is in the carrier $\Phi$ of $I$. Conversely, any homomorphism $\phi$ in $\Phi$ is $|\cdot|_{I}$-continuous on $A$ and hence uniformly continuous. Therefore, $\phi$ has a unique continuous extension $\bar{\phi}$ to $\bar{A}$. Clearly this extension is a homomorphism in $\Psi$. But any homomorphic extension of $\phi$ to all of $\bar{A}$ is continuous (as shown at the beginning of this proof) and must therefore, coincide with $\bar{\phi}$. Thus $\bar{\phi} \rightarrow \bar{\phi} \mid A$ is a biunique map from $\Psi$ onto $\Phi$. It is clearly continuous, so, since $\Psi$ and $\Phi$ are compact Hausdorff spaces, it must be a homeomorphism. This demonstrates the necessary identification and finishes the proof.

Because of the radical-like properties of the ideal $I^{\prime}$, it is possible to use Theorem $B$ to extract information about general convex intervals - even when they are unbounded. For this end, another proposition is required.

Proposition 6. Suppose $I$ is a convex interval that generates an algebra $A$, and let $J$ denote the canonical image of $I$ in the quotient algebra $A / I^{\prime}$. Then

(a) $J^{\prime}=0$,

(b) the linear closure $\bar{I}$ of $I$ is again a convex interval,

(c) if $I$ is linearly closed and $x \in I$ and $t \in I^{\prime}$, then $x+t$ is in $I$.

Proof. These assertions are either meaningless or trivial if $I^{\prime}=A$, so we proceed with the extra assumption that $I^{\prime}$ is a proper ideal.

(a) Suppose $p \in A$ maps into $J^{\prime}$ under the quotient homomor- 
phism. Then for each integer $n>0$, there is some $x_{n} \in I$ such that $1 / 2+2 n p \equiv x_{n} \bmod I^{\prime}$. That is, for some $t_{n}$ in $I^{\prime}, 1 / 2+2 n p-t_{n}=x_{n}$. Thus $1 / 2+n p=(1 / 2) x_{n}+1 / 2\left(1 / 2+t_{n}\right)$. Since $t_{n} \in I^{\prime}$, this last expression is the average of points in $I$ and hence lies in $I$. Whence $p \in I^{\prime}$, so $p \equiv 0 \bmod I^{\prime}$. Thus $J^{\prime}=0$.

(b) Topologize $A$ by taking as a basis of neighborhoods at 0 , the family of convex sets radial at 0 . This makes $A$ into a topological vector space [13, p. 53]. By Proposition $3 b, I$ is radial from $1 / 2$ and hence has nonvoid interior in this topology. Therefore, the topological closure coincides with the linear closure $\bar{I}$. Now the topology on $A$ is so strong that all linear maps from $A$ into $A$ are continuous. In particular, for any fixed $a \in A$, the maps $x \rightarrow x a$ and $x \rightarrow a x$ are continuous. Appealing to Lemma A with $X=A$ and $Y=I$, we my now conclude that $\bar{I}$ is a semigroup; the other interval properties are also easily verified.

(c) For any scalar $\lambda$ in (0: 1$), \lambda(x+t)+(1-\lambda) 1 / 2=\lambda x+$ $(1-\lambda)(1 / 2+\lambda /(1-\lambda) t)$, which is in $I$. Thus $x+t$ is the endpoint of a segment whose interior lies entirely in $I$. If $I$ is linearly closed, this forces $x+t$ to be in $I$.

THEOREM C. Let $S$ be a subset of an algebra $A$ which generates $A$ as an algebra. Let $I$ be the smallest convex interval in $A$ which contains $S$. If $\bar{I}$ denotes the linear closure of $I$, and core $(I)$ denotes the set of all points in $I$ from which $I$ is radial, and $\Phi$ denotes the carrier of $S$ in $A$, then

(a) $\bar{I}=\{x \in A: 0 \leqq \phi(x) \leqq 1$ for all $\phi$ in $\Phi\}$,

(b) core $(I)=\{x \in A: 0<\phi(x)<1$ for all $\phi$ in $\Phi\}$,

(c) $I^{\prime}=\{x \in A: \phi(x)=0$ for all $\phi$ in $\Phi\}$.

Proof. If $I^{\prime}=A$, then $\Phi$ is empty and the above equalities hold by default. Thus we suppose $I^{\prime}$ is a proper subset of $A$. Since the core of any convex set is the same as the core of its linear closure $[18$, p. 11], core $(I)=$ core $(\bar{I})$. Knowing this and Proposition $3 b$, one can give a simple argument to show $I^{\prime}=(\bar{I})^{\prime}$. Thus we can concentrate on $\bar{I}$, which by Proposition $6 \mathrm{~b}$ is also an interval.

Let $J$ be the image of $\bar{I}$ in the quotient algebra $A / I^{\prime}$. We claim $J$ is linearly closed. For suppose $p+I^{\prime}$ is the endpoint of some segment whose interior lies in $J$. Let $q+I^{\prime}$ be the other endpoint. Then for each $\lambda$ in $(0: 1)$, there is some $x_{\lambda}$ in $\bar{I}$ such that $\lambda p+(1-\lambda) q \equiv x_{\lambda} \bmod I^{\prime}$. That is, there is some $t_{\lambda}$ in $I^{\prime}$ with $\lambda p+$ $(1-\lambda) q=x_{\lambda}+t_{\lambda}$. By Proposition 6c, the points $x_{\lambda}+t_{\lambda}$ lie in $\bar{I}$. Thus the interior of the segment from $p$ to $q$ lies in $\bar{I}$, so $p \in \bar{I}$. 
Combining this with Proposition 6a, we can conclude that $J$ is a linearly closed and bounded interval and then apply Theorem B. Let $\Psi$ denote the carrier of $J$ in $A / I^{\prime}$. If $x$ is a point of $A$ not in $I^{\prime}$, then $x+I^{\prime}$ is nonzero in $A / I^{\prime}$. Thus by Theorem $\mathrm{B}$, there is some $\psi$ in $\Psi$ with $\psi\left(x+I^{\prime}\right) \neq 0$. We can lift $\psi$ to a homomorphism in $\Phi$ which does not vanish at $x$ simply be composing $\psi$ with the canonical map of $A$ onto $A / I^{\prime}$. Thus (c) if proved.

Since the set described on the right in (a) above is clearly a linearly closed convex interval containing $S$, to prove equality it suffices to show that $\bar{I}$ contains this set. If $x$ is a point of $A$ not in $\bar{I}$, then $x+I^{\prime}$ cannot belong to $J$. For otherwise, there would be a $y$ in $\bar{I}$ with $x \equiv y \bmod I^{\prime}$ and thus, for some $t$ in $I^{\prime}, x=y+t$. By Proposition 6c, this would force $x$ to be in $\bar{I}$. Thus $x+I^{\prime}$ is not in $J$ so by Theorem B there is some $\psi$ in $\Psi$ that maps $x+I^{\prime}$ into a number not in [0:1]. Again $\psi$ may be lifted to a homomorphism $\phi$ in $\Phi$ such that $\phi(x)=\psi(x+I) \notin[0: 1]$. Whence (a) is established.

If $x$ is a core point of $\bar{I}$, then for some $\lambda>0, \bar{I}$ contains $x+\lambda \cdot 1$, so, by (a), $\phi(x)=\phi(x+\lambda)-\phi(\lambda) \leqq 1-\lambda<1$ for any $\phi$ in $\Phi$. Similarly $\phi(x)>0$ for all $\phi$ in $\Phi$. Thus the core of $\bar{I}$ is contained in the right-hand set of (b). Let us show the reverse inclusion. Since $\Phi$ is compact in the topology of pointwise convergence on $A$, if $\phi(x)<1$ for all $\phi$ in $\Phi$, then for some $\mu>0, \phi(x) \leqq 1-\mu$ for all $\phi$ in $\Phi$. Similarly, if $\phi(x)>0$ for all $\phi$ in $\Phi$, then for some $\lambda>0, \phi(x) \geqq \lambda$. Now let $\theta=\min (\lambda, \mu)$ and let $y=(1-\theta)^{-1}(x-\theta / 2)$. For any $\phi$ in $\Phi, \phi(x-\theta / 2) \geqq 0$ since $\theta \leqq \lambda$. Dividing by $1-\theta \geqq 0$, we get $\phi(y) \geqq 0$. Also, $\phi(x-\theta / 2) \leqq \phi(x) \leqq 1-\theta$ since $\theta \leqq \mu$. On division by $1-\theta$, this yields $\phi(y) \leqq 1$. Therefore, by part (a), $y$ must belong to $\bar{I}$. Since $x=(1-\theta) y+\theta \cdot 1 / 2$ expresses $x$ as an interior point of the segment from the core point $1 / 2$ of $\bar{I}$ to the point $y$ of $\bar{I}, x$ must all belong to the core of $\bar{I}$. Whence, (b) is established and the proof is completed.

Let us now turn our attention to some consequences of the theorems proved above.

Proof of Stone's algebra theorem. In the archimedean ordered algebra $A$, let $I=\{x \in A: 0 \leqq x \leqq 1\}$. Because 1 is an order unit, the algebra $A$ is generated by $I$. Because $A$ is archimedean, $I$ is linearly closed and $I^{\prime}=0$. Thus Stone's theorem follows at once from Theorem $B$.

We now prove a slightly strengthened version of a result of Dai and DeMarr [2, p. 651].

THEOREM. Suppose $A$ is an archimedean ordered algebra such 
that for any $x \geqq 0,1+x$ has a positive inverse. If every element of $A$ is the difference of positive elements, then multiplication in $A$ is commutative.

Proof. Define an interval $I$ as above. Again $I$ is linearly closed and $I^{\prime}=0$, but possibly the algebra $A(I)$ generated by $I$ is smaller than $A$. In any case, $A(I)$ is commutative (by Proposition 4a). For any $x \geqq 0$, by hypothesis $(1+x)^{-1}$ is positive and hence is also less than 1. Thus for any two positive elements $x$ and $y,(1+x)^{-1}$ and $(1+y)^{-1}$ belong to $I$ and hence commute. It follows that $1+x$ and $1+y$ commute, so $x y=y x$. By hypothesis, $A$ is generated by its positive cone, so $A$ is commutative.

Dai and DeMarr assumed that every decreasing sequence bounded below had an infimum. When every element is the difference of positive elements, this implies the archimedean property. For suppose $a \geqq 0$. Then $a / n$ is a decreasing sequence bounded below by 0 , and it therefore has an infimum $p \geqq 0$. Since $a /(2 n) \geqq p$ for all $n$, multiplying by 2 yields $a / n \geqq 2 p$ for all $n$. Hence $p \geqq 2 p$, so $p=0$. Now if $n x \leqq y$ for all $n$, write $y=a-b$ where $a$ and $b$ are positive to get $n x \leqq a$ for all $n$. Then $a / n-x$ is a decreasing sequence of positive elements and hence has an infimum $q \geqq 0$. Clearly, $q+x$ is the infimum of $a / n$, but we saw that infimum must be zero. Hence $x=-q \leqq 0$.

Let us now give proofs for the two theorems stated at the beginning of this paper.

Proof of Theorem 1. We shall show that, with our restriction on $M$, the interval in Example (III) satisfies the hypotheses of Proposition 4a. It takes only a routine check to show that this interval is convex and linearly closed. Establishing the necessary boundedness also proceeds easily. Suppose that $1 / 2+n Q$ belongs to the interval for all positive integers $n$. To show that $Q$ is identically zero on $M$, it suffices, by the assumption on $M$, to show that $Q$ sends every nonnegative function $f$ in $M$ to 0 . But for each positive integer $n, 0 \leqq(1 / 2) f+n Q(f) \leqq f$, so for each $x$ in $X$,

$$
-\frac{1}{2 n} f(x) \leqq Q(f)(x) \leqq \frac{1}{2 n} f(x) .
$$

Whence $Q(f)=0$.

One could strengthen the conclusion of Theorem 1 by applying Theorem B instead of Proposition 4a to the interval of operators in Example (III). In general there is no clear relationship between the compact Hausdorff space $\Phi$ on which the operators can be represented as continuous functions and the set $X$. But in the case that $M$ is 
the space $C(X)$ of all continuous functions on compact Hausdorff $X$, it is easy to show directly that these operators have a particularly simple form. Namely, for any such $T$ there is a $g$ in $C(X)$ with $0 \leqq g(x) \leqq 1$ for all $x$ such that $T(f)=g \cdot f$ for all $f$ in $C(X)$ (use the results of [3, p. 490] or apply an argument like [11, p. 628]).

Proof of Theorem 2., Let $S=\left\{a_{n} x^{n}\right\}_{n=1}^{\infty} \cup\{1\}$. Since $a_{1}>0$, the algebra generated by $S$ is clearly the algebra $R[x]$ of all real polynomials. Let us identify the carrier $\Phi$ of $S$. Any homomorphism of $\boldsymbol{R}[x]$ into $\boldsymbol{R}$ is given by evaluation at some point $\lambda$ in $\boldsymbol{R}$. Thus $\Phi$ can be regarded as the set of all $\lambda \in \boldsymbol{R}$ such that $0 \leqq a_{n} \lambda^{n} \leqq 1$ for all $n$. Thus $\lambda \geqq 0$ and $\lambda \leqq \inf \left(1 / a_{n}\right)^{1 / n}$. By hypothesis, this infimum is 0 . Therefore, the only homomorphism in $\Phi$ is evaluation at 0 . From this, the conclusion of Theorem 2 can be read off directly from Theorem C, part (b).

It is well-known that the topology of a compact Hausdorff space $X$ can be recovered from the algebraic structure of $C(X)$. A usual procedure is to identify the ideals of $C(X)$ with closed sets in the topology [6]. But, unfortunately, this is not a biunique correspondence. The next theorem shows another procedure for recovering the topology of $X$ from $C(X)$.

TheOREM 3. Let X be a compact Hausdorff space. For each closed subset $K$ of $X$, let $I(K)$ denote the set of all continuous functions on $X$ which take only values between 0 and 1 on $K$. Then

$$
K \longleftrightarrow I(K)
$$

is a one-to-one correspondence between the closed subsets of $X$ and the linearly closed convex intervals in $C(X)$ which generate $C(X)$.

Proof. If $I$ is a linearly closed convex interval generating $C(X)$, we need to show that $I=I(K)$ for some $K$. Since $X$ is compact, any homomorphism of $C(X)$ into $R$ is evaluation at some point of $X$. Thus the carrier $\Phi$ of $I$ may be regarded as a subset of $X$. Namely, $\Phi=\{x \in X: 0 \leqq f(x) \leqq 1$ for all $f$ in $I\}$. Since the functions $f$ are continuous, $\Phi$ is closed. Applying Theorem C, part a, we get $I=I(\Phi)$. Thus $K \rightarrow I(K)$ is surjective. That it is also one-to-one follows immediately from Urysohn's lemma.

Theorem 4. Suppose that $A \neq \boldsymbol{R}$ is a simple $\boldsymbol{R}$-algebra. Then any set $S$ which generates $A$ as an $\boldsymbol{R}$-algebra generates $A$ as a convex interval.

Proof. Since $A$ is simple and $A \neq \boldsymbol{R}$, there can be no algebra 
homomorphisms from $A$ to $R$. Thus the carrier $\Phi$ of $S$ is empty. The theorem now follows from Theorem $C$.

In essence this result asserts that if any element of a simple algebra can be obtained from $S$ via the operations of multiplication and real linear combination, then any element is obtainable from $S$ by means of multiplication, subtraction from 1 , and convex combination. An analogue of this result for algebras over more general fields might prove useful in the study of $p$-adic number fields and other locally compact fields. Unfortunately, no such analogues are presently known.

A related direction for improvement would be the weakening of the convexity assumption. Although in this paper convexity plays a major role in the arguments, Jewett's proof of his theorem does not involve convexity. It does rely, however, on the very potent hypothesis of uniform closure. Is it possible to simultaneously refine Theorem $\mathrm{C}$ and Jewett's theorem, giving a precise description of the interval (not necessarily closed or convex) generated by an arbitrary set $S$ ?

Also, the reader may have noticed that occasionally the full strength of the interval conditions was not required. Often it sufficed to note that an interval was closed under the operation $x \circ y=x+$ $y-x y=1-(1-x)(1-y)$. Using this observation, can one obtain a sizable portion of the above theory for algebras without 1 (in particular, the group algebras)?

We shall close with a curious logical conundrum which arises from the following algebraic consequence of the main theorems.

THEOREM 5. If I is a convex interval in an algebra $A$, then, for any $x$ and $y$ in $I, x y-y x$ belongs to $I^{\prime}=\{t \in A: 1 / 2+n t \in I$ for all integers $n\}$.

Proof. We may assume without loss of generality that $I$ generates $A$. Since $\boldsymbol{R}$ is commutative, any homomorphism from $A$ into $\boldsymbol{R}$ vanishes on all commutators $x y-y x$. The theorem now follows from Theorem C.

This theorem is remarkable in that it is a purely algebraic fact which seemingly requires a rather involved analytic proof. There are other results of this kind known - in example, the invertibility of elements in certain group algebras [12, p. 122]. But a more remarkable aspect of Theorem 5 is that it implies, in some sense, that an algebraic proof can be found! The reasoning for this follows.

Let $A(x, y)$ be the free algebra with 1 generated by two noncommutative variables. If $J$ is the smallest interval in $A$ containing $x$ and $y$, then $J$ may be inductively constructed as follows: 


$$
\begin{aligned}
J_{0} & =\{0,1, x, y\} \\
J_{n+1} & =\left\{1-a b, a b: a \text { and } b \text { are in } J_{n}\right\} \\
J & =\bigcup_{n=0}^{\infty} J_{n} .
\end{aligned}
$$

Applying Theorem 5 to the convex interval $I=\operatorname{conv} J$, we must have $x y-y x$ in $I^{\prime}$. Thus for each integer $k$ there is some formula involving $x, y$, convex combinations, and interval operations for $1 / 2+$ $k(x y-y x)$. Being able to describe these formulae explicitly would constitute a proof of Theorem 5. The long route presented above is evidence that this investigator has tried in vain to produce the formulae. Surprisingly, for any fixed $k$, there does "exist" a finite algorithm for determining the kth formula. Since $1 / 2+k x y-k y x$ has rational coefficients, it must be a rational convex combination of points in $J$. (Let $S$ be a subset of $J$ with as few elements as possible such that $1 / 2+k(x y-y x) \in \operatorname{conv} S$. There is then a unique convex combination of the points of $S$ yielding $1 / 2+k(x y-y x)$. This can be interpreted as the unique solution to an appropriate set of linear equations, all having rational coefficients.) If we let $H_{n}$ be the set of all convex combinations, using only rational numbers whose denominators are less than $n$, of points in $J_{n}$, then $1 / 2+k(x y-y x)$ must belong to some $H_{n(k)}$. Each $H_{n}$ is finite and can be determined in a finite, albeit large, number of steps. Hence after a finitely terminating search through the $H_{n}$ 's, one must eventually discover $1 / 2+k(x y-y x)$ and thereby discover the formula which puts it in $I$.

The search even for $k=1$ has proved futile. The best result is the trivial formula:

$$
\frac{1}{2}+\frac{1}{2}(x y-y x)=\frac{1}{2}(1-y x)+\frac{1}{2}(x y) .
$$

Could it be that the existence of these formulae is logically dependent on the axiom choice and that they "really are not there?"

The author wishes to express his gratitude to Professor Isaac Namioka for many enlightening conversations about vector space topologies. His comments frequently assisted the author to a better understanding and presentation of several of the arguments in this paper.

\section{REFERENCES}

1. W. G. Bade, The Banach space $C(S)$, Arhus University Lecture Note Series, No. 26, 1971.

2. T. Dai, On some special classes of partially ordered linear algebras, J. of Math. Anal. and Appl., 40 (1972), 649-682. 
3. N. Dunford and J. T. Schwartz, Linear Operators (I), Interscience, New York, 1966.

4. J. Eckhoff, Der Satz von Randon in konvexen Productstrulturen I-II, Monatsh. Math., 72 (1968), 303-314; 73 (1969), 7-30.

5. L. Fuchs, Partially Ordered Algebraic Systems, Pergamon Press, Oxford, 1963.

6. L. Gillman and M. Jerison, Rings of Continuous Functions, Van Nostrand, Princeton, 1960.

7. E. Hewitt and K. Stromberg, Real and Abstract Analysis, Springer-Verlag, New York, 1969.

8. D. Hilbert, Grundlagen der Geometrie, B. G. Teubner, Stuttgart, 1968.

9. R. I. Jewett, A variation on the Stone-Weierstrass theorem, Amer. Math. Soc., Proceedings, 14 (1963), 690-693.

10. R. V. Kadison, A Representation Theorem for Commutative Topological Algebras, Amer. Math. Soc. Memoirs, \#7, 1951.

11. I. Kaplansky, Lattices of continuous functions II, Amer. J. Math., 70 (1948), 626-634.

12. - Fields and Rings, Univ. of Chicago Press, Chicago, 1969.

13. J. Kelly and I. Namioka, Linear Topological Spaces, Van Nostrand, Princeton, 1963.

14. J. R. Reay, Caratheodory theorems in convex product structures, Pacific J. Math., 35 (1970), 227-230.

15. C. Rickart, Banach Algebras, Van Nostrand, Princeton, 1963.

16. H. H. Schaefer, Topological Vector Spaces, Springer-Verlag, New York, 1971.

17. M. H. Stone, A general theory of spectra I, PNAS USA, 26 (1940), 280-283.

18. F. A. Valentine, Convex Sets, McGraw-Hill, New York, 1964.

19. J. von Neumann, Probabilistic logics and the synthesis of organisms from unrealiable components, Automata Studies, Princeton Univ. Press, Princeton, 1956.

Received July 5, 1972 and in revised form January 28, 1974. The author was supported by a NSF Graduate Fellowship during the preparation of this paper.

UNIVERSITY OF WASHINGTON

Current address: Louisiana State University 



\section{PACIFIC JOURNAL OF MATHEMATICS}

\section{EDITORS}

RICHARD ARENS (Managing Editor)

University of California

Los Angeles, California 90024

\section{J. DUGUNDJI}

Department of Mathematics University of Southern California Los Angeles, California 90007

D. Gilbarg and J. Milgram

Stanford University

Stanford, California 94305

University of Washington
Seattle, Washington 98105

ASSOCIATE EDITORS
E. F, BECKENBACH
B. H. NEUMANN
F. WOLF
K. Yoshida

\section{SUPPORTING INSTITUTIONS}

\author{
UNIVERSITY OF BRITISH COLUMBIA \\ CALIFORNIA INSTITUTE OF TECHNOLOGY \\ UNIVERSITY OF CALIFORNIA \\ MONTANA STATE UNIVERSITY \\ UNIVERSITY OF NEVADA \\ NEW MEXICO STATE UNIVERSITY \\ OREGON STATE UNIVERSITY \\ UNIVERSITY OF OREGON \\ OSAKA UNIVERSITY
}

\author{
UNIVERSITY OF SOUTHERN CALIFORNIA \\ STANFORD UNIVERSITY \\ UNIVERSITY OF TOKYO \\ UNIVERSITY OF UTAH \\ WASHINGTON STATE UNIVERSITY \\ UNIVERSITY OF WASHINGTON \\ * * * * \\ AMERICAN MATHEMATICAL SOCIETY \\ NAVAL WEAPONS CENTER
}

The Supporting Institutions listed above contribute to the cost of publication of this Journal, but they are not owners or publishers and have no responsibility for its content or policies.

Mathematical papers intended for publication in the Pacific Journal of Mathematics should be in typed form or offset-reproduced, (not dittoed), double spaced with large margins. Underline Greek letters in red, German in green, and script in blue. The first paragraph or two must be capable of being used separately as a synopsis of the entire paper. Items of the bibliography should not be cited there unless absolutely necessary, in which case they must be identified by author and Journal, rather than by item number. Manuscripts, in duplicate if possible, may be sent to any one of the four editors. Please classify according to the scheme of Math. Rev. Index to Vol. 39. All other communications to the editors should be addressed to the managing editor, or Elaine Barth, University of California, Los Angeles, California, 90024.

100 reprints are provided free for each article, only if page charges have been substantially paid. Additional copies may be obtained at cost in multiples of 50 .

The Pacific of Journal Mathematics is issued monthly as of January 1966. Regular subscription rate: $\$ 72.00$ a year (6 Vols., 12 issues). Special rate: $\$ 36.00$ a year to individual members of supporting institutions.

Subscriptions, orders for back numbers, and changes of address should be sent to Pacific Journal of Mathematics, 103 Highland Boulevard, Berkeley, California, 94708.

PUBLISHED BY PACIFIC JOURNAL OF MATHEMATICS, A NON-PROFIT CORPORATION

Printed at Kokusai Bunken Insatsusha (International Academic Printing Co., Ltd.), 270, 3-chome Totsuka-cho, Shinjuku-ku, Tokyo 160, Japan.

Copyright (C) 1973 by Pacific Journal of Mathematics Manufactured and first issued in Japan 


\section{Pacific Journal of Mathematics}

\section{Vol. 53, No. $2 \quad$ April, 1974}

Kenneth Abernethy, On characterizing certain classses of first countable spaces by

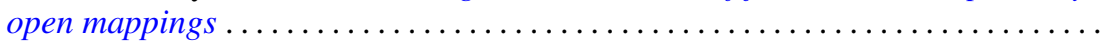

Ross A. Beaumont and Donald Lawver, Strongly semisimple abelian groups .......

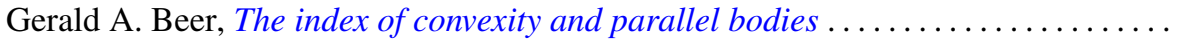

Victor P. Camillo and Kent Ralph Fuller, On Loewy length of rings ..............

Stephen LaVern Campbell, Linear operators for which $T^{*} T$ and $T T^{*}$ commute.

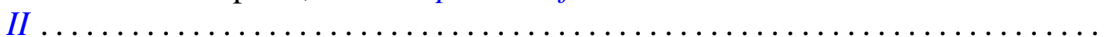

Charles Kam-Tai Chui and Philip Wesley Smith, Characterization of a function by

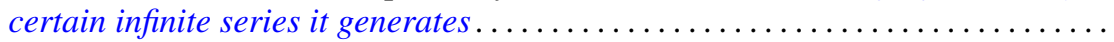

Allan L. Edelson, Conjugations on stably almost complex manifolds . ...........

Patrick John Fleury, Hollow modules and local endomorphism rings . . ..........

Jack Tilden Goodykoontz, Jr., Connectedness im kleinen and local connectedness in

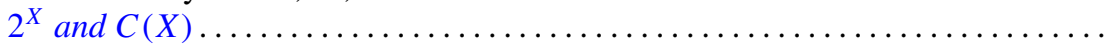

Robert Edward Jamison, II, Functional representation of algebraic intervals .......

Athanassios G. Kartsatos, Nonzero solutions to boundary value problems for

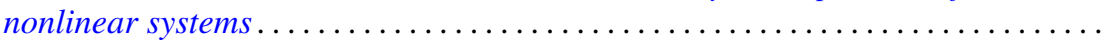

Soon-Kyu Kim, Dennis McGavran and Jingyal Pak, Torus group actions on simply

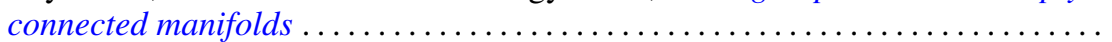

David Anthony Klarner and R. Rado, Arithmetic properties of certain recursively

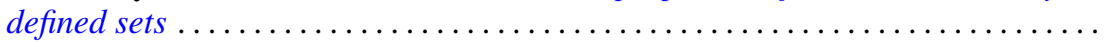

Ray Alden Kunze, On the Frobenius reciprocity theorem for square-integrable

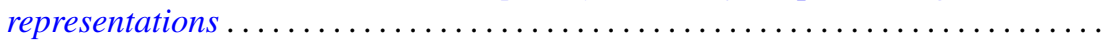

John Lagnese, Existence, uniqueness and limiting behavior of solutions of a class of differential equations in Banach space...

Teck Cheong Lim, A fixed point theorem for families on nonexpansive mappings Lewis Lum, A quasi order characterization of smooth continua

Andy R. Magid, Principal homogeneous spaces and Galois extensions . .

Charles Alan McCarthy, The norm of a certain derivation ..... . .

Louise Elizabeth Moser, On the impossibility of obtaining $S^{2} \times S^{1}$ by elementary surgery along a knot. .

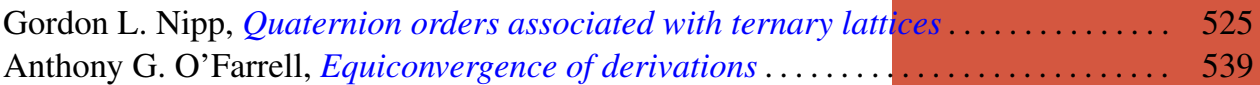

Dorte Olesen, Derivations of $A W^{*}$-algebras are inner . . . . . . . . . . . . . . . 555

Dorte Olesen and Gert Kjærgaard Pedersen, Derivations of $C^{*}$-algebras have

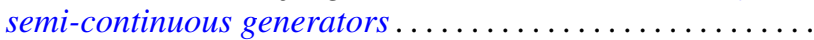

Duane O’Neill, On conjugation cobordism.

Chull Park and S. R. Paranjape, Probabilities of Wiener paths crossing differentiable

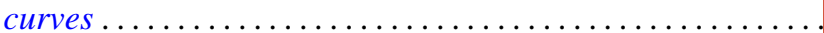

Edward Ralph Rozema, Almost Chebyshev subspaces of $L^{1}(\mu$;

Lesley Millman Sibner and Robert Jules Sibner, A note on the Atiyah-Bott fixed

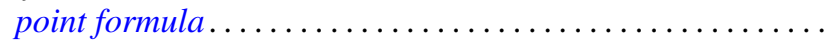

Betty Salzberg Stark, Irreducible subgroups of orthogonal groups generated by

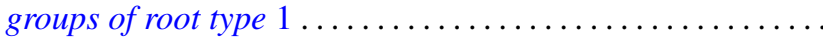

N. Stavrakas, A note on starshaped sets, $(k)$-extreme points and the half ray

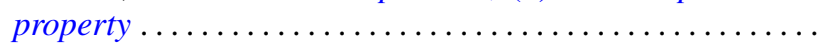

Carl E. Swenson, Direct sum subset decompositions of $Z \ldots \ldots$ 